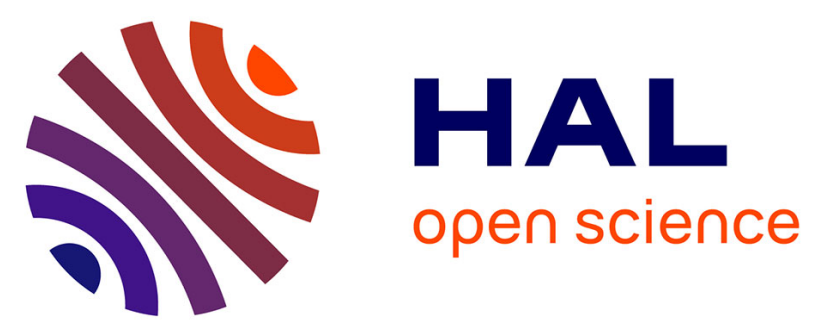

\title{
Analysis of Radiation Damage in Light Water Reactors: Comparison of Cluster Analysis Methods for the Analysis of Atom Probe Data
}

Jonathan Hyde, Gérald da Costa, Constantinos Hatzoglou, Hannah Weekes, Bertrand Radiguet, Paul Styman, François Vurpillot, Cristelle Pareige, Auriane Etienne, Giovanni Bonny, et al.

\section{To cite this version:}

Jonathan Hyde, Gérald da Costa, Constantinos Hatzoglou, Hannah Weekes, Bertrand Radiguet, et al.. Analysis of Radiation Damage in Light Water Reactors: Comparison of Cluster Analysis Methods for the Analysis of Atom Probe Data. Microscopy and Microanalysis, 2017, 23 (02), pp.366 - 375. 10.1017/s1431927616012678 . hal-01765920

\section{HAL Id: hal-01765920 \\ https://hal.science/hal-01765920}

Submitted on 29 May 2019

HAL is a multi-disciplinary open access archive for the deposit and dissemination of scientific research documents, whether they are published or not. The documents may come from teaching and research institutions in France or abroad, or from public or private research centers.
L'archive ouverte pluridisciplinaire HAL, est destinée au dépôt et à la diffusion de documents scientifiques de niveau recherche, publiés ou non, émanant des établissements d'enseignement et de recherche français ou étrangers, des laboratoires publics ou privés. 


\title{
Analysis of Radiation Damage in Light Water Reactors: Comparison of Cluster Analysis Methods for the Analysis of Atom Probe Data
}

\author{
Jonathan M. Hyde, ${ }^{1,2}$ Gérald DaCosta, ${ }^{3}$ Constantinos Hatzoglou, ${ }^{3}$ Hannah Weekes, ${ }^{1}$ \\ Bertrand Radiguet, ${ }^{3}$ Paul D. Styman, ${ }^{1,2, *}$ Francois Vurpillot, ${ }^{3}$ Cristelle Pareige, ${ }^{3}$ Auriane Etienne, ${ }^{3}$ \\ Giovanni Bonny, ${ }^{4}$ Nicolas Castin, ${ }^{4}$ Lorenzo Malerba, ${ }^{4}$ and Philippe Pareige ${ }^{3}$ \\ ${ }^{1}$ National Nuclear Laboratory, Culham Science Centre, Building D5, Abingdon, Oxfordshire OX14 3DB, UK \\ ${ }^{2}$ Department of Materials, University of Oxford, Parks Road, Oxford OX1 3PH, UK \\ ${ }^{3}$ UNIROUEN, INSA Rouen, CNRS, Groupe de Physique des Matériaux, Normandie Université, 76000 Rouen, France \\ ${ }^{4}$ Studiecentrum voor Kernenergie-Centre d'Etudes de l'Energie Nucléaire (SCK-CEN), Institute of Nuclear Materials Science, \\ Expert Group of Structural Materials, Boeretang 200, B-2400 Mol, Belgium
}

\begin{abstract}
Irradiation of reactor pressure vessel (RPV) steels causes the formation of nanoscale microstructural features (termed radiation damage), which affect the mechanical properties of the vessel. A key tool for characterizing these nanoscale features is atom probe tomography (APT), due to its high spatial resolution and the ability to identify different chemical species in three dimensions. Microstructural observations using APT can underpin development of a mechanistic understanding of defect formation. However, with atom probe analyses there are currently multiple methods for analyzing the data. This can result in inconsistencies between results obtained from different researchers and unnecessary scatter when combining data from multiple sources. This makes interpretation of results more complex and calibration of radiation damage models challenging. In this work simulations of a range of different microstructures are used to directly compare different cluster analysis algorithms and identify their strengths and weaknesses.
\end{abstract}

Key words: atom probe field ion microscopy, statistical analysis, solute clustering

\section{INTRODUCTION}

Most of the operating nuclear reactors in the world are pressurized water reactors (PWRs). In a PWR, the reactor pressure vessel (RPV) is the second barrier between the fuel and the outside world. They are made of low alloyed bainitic steels (NiMoCr, NiMoCrV, A-533B). During service, neutrons produced in the reactor core generate displacement damage, resulting in a supersaturation of vacancies and selfinterstitial atoms (SIAs). These supersaturated point defects (PDs) can agglomerate to form extended defects, but also enhance and modify solute diffusion causing solute redistribution in the material. This irradiation ageing is responsible for hardening and nonhardening embrittlement of RPV steels and the degradation can be life limiting for nuclear reactors. As irradiation damage occurs at the nm-scale, atom probe tomography (APT) (Miller et al., 1996; Miller, 2000; Gault et al., 2012) is a suitable tool to characterize irradiation-induced nanofeatures in terms of their nature, chemical composition, size, shape, and number density.

Small clusters or precipitates containing $\mathrm{Cu}, \mathrm{Mn}, \mathrm{Ni}, \mathrm{Si}$, and $\mathrm{P}$ are often observed using APT (Pareige et al., 1997; Miller et al., 2000, 2007; Carter et al., 2001; Radiguet et al., 2009; Huang et al., 2014). The chemical composition of these clusters, and their evolution with neutron fluence, depends on many variables including bulk composition and detailed irradiation conditions (e.g., temperature and flux). However, the reported chemical compositions also depend on artifacts inherent to APT, the methodologies used to characterize the APT data and the specific scientists involved in the work (e.g., through the choice of analysis parameters). The result has been a plethora of inconsistent nomenclatures. For instance, the terms copper rich precipitates, copper enriched clusters, manganese nickel silicon precipitates, manganese nickel precipitates, nickel silicon precipitates have been used interchangeably by different researchers (Auger et al., 1995; Odette, 1995; Miller \& Russell, 2007; Pareige et al., 1997; Carter et al., 2001; Takeuchi et al., 2010; Wells et al., 2014).

A consistent and robust approach to data analyses (and also nomenclature) is necessary to enable direct comparison of APT data obtained in different laboratories, whereas enabling modelers to analyze the outcome of atomistic simulations consistently with experiments, allowing direct and fair comparison. There are three issues to address. First, it is necessary to assess the intrinsic limitations of APT techniques, so that the relationship between microstructure observed using APT and the actual microstructure is understood. Second it is necessary to ensure that the cluster analysis algorithms provide an accurate and robust description of the irradiation-induced microstructural features. Third, it is necessary to agree on protocols to be used for the analysis of, especially, atomistic model results, so as to enable a fair comparison with APT results. 
There are several known limitations of APT, including concerns that the reconstruction algorithms used are too simplistic. For instance, for the analysis of RPV steels, one major concern is that trajectory aberrations, due to difference of the evaporation field between the solute clusters and the surrounding matrix, could bias their measured chemical composition and structure. Other concerns, include (i) the fact that APT cannot directly detect the presence of vacancies, (ii) the spatial resolution is imperfect, (iii) the detection efficiency is imperfect, and (iv) the very small analyzed volume may not be fully representative of a material as complex as a steel (Miller et al., 1996; Miller, 2000; Gault et al., 2012).

Demonstrating that cluster detection algorithms accurately characterize the multitude of microstructural features observed in RPV steels is also challenging. The very small size of these solute clusters and their apparently dilute nature makes their identification, inside three-dimensional (3D) data sets containing tens or hundreds of millions of atoms, very challenging. The very small size of the features means that a relatively high fraction of the associated atoms are interface atoms, and thus their measured sizes, number density, and compositions can also strongly depend on the algorithms used to detect them. Currently, there is no agreement on what constitutes the most "appropriate" analysis methodology (Marquis \& Hyde, 2010). Furthermore, all methods require the use of user-defined parameters and their selection is not trivial. Methods based on the maximum separation method (MSM) (Hyde \& English, 2001; Heinrich et al., 2003; Kolli \& Seidman, 2007; Morley et al., 2009; Styman et al., 2013) are most widely used, but there are many variants and options at each stage of the process, including defining the chemical identities of core atoms, selecting an appropriate maximum separation distance, identifying which noncore atoms also belong to each cluster and whether to apply an erosion step to minimize interface effects. Other methods, based on concentration threshold criteria, have also been developed and used (Lefebvre et al., 2016) by several groups. As for MSMs, the definition of the chemical identities and the selection of appropriate parameters is an important step to get quantitative and reliable results.

In this work, simulations have been used to create representative APT data sets with known microstructures. The microstructural data have been degraded to simulate the limitations of APT and the resulting data have been analyzed blind using two analysis methodologies. The results have been compared with each other and the strengths and weaknesses of the methods assessed. This builds on previous work by Hyde et al. (2011) by using multiple analyses methods, and adding the effect of local magnification.

\section{Methodology}

\section{Simulated Microstructures}

In all, six simulated microstructures, each $60 \times 60 \times 60 \mathrm{~nm}^{3}$, of a simple ferritic alloy were created (bcc lattice with a lattice parameter of $0.28 \mathrm{~nm}$ ). The simulated microstructures were populated with clusters with a number density of between 5 and $10 \times 10^{23} \mathrm{~m}^{-3}$. Three different cluster sizes were chosen (approximate radii of $\sim 0.5,1.0$, and $1.5 \mathrm{~nm}$ ) with each cluster having a solute content of either $\sim 50$ or $100 \%$. Thus each simulated microstructure contained multiple clusters of different sizes and compositions ( 18 of each radius and composition combination). A bulk alloy concentration of $\sim 1 \%$ was chosen. The simulated microstructures also took into account experimental artifacts. In three data files, the imperfect detection efficiency of the CAMECA LEAP 3000 or $4000 \mathrm{HR}$ was simulated by randomly removing $60 \%$ of the atoms. In four of the simulations, uncertainties in the atom positions were modeled by adding a Gaussian scatter to the coordinates $(2 \sigma=0.5$ or $1 \mathrm{~nm}$ for the $X$ and $Y$ coordinates, and $2 \sigma=0.1 \mathrm{~nm}$ for the $Z$ coordinates). The resulting matrix of simulated microstructures is summarized in Table 1 . In addition, three of the simulations were further degraded by modeling the compression of atoms in reconstructed APT data associated with the local magnification effect (only simulation 6 was analyzed here, yielding a total of seven datasets for analysis). The compression algorithm used is detailed in the next section. The combinations were designed to enable the influence of experimental artifacts on the detectability and characterization of clusters to be assessed.

A further nine simulated microstructures were generated for an alloy with a bulk solute concentration of $4 \%$ and then a further 12 simulated microstructures were created to model core shell cluster structures. The analysis of these additional simulations will be the subject of a future paper.

Table 1. Simulated Microstructures and Simulations of Experimental Artifacts.

\begin{tabular}{|c|c|c|c|c|c|}
\hline $\begin{array}{l}\text { Simulation } \\
\text { no. }\end{array}$ & $\begin{array}{c}\text { Cluster } \\
\text { Radius (nm) }\end{array}$ & $\begin{array}{c}\text { Cluster Solute } \\
\text { Concentrations (\%) }\end{array}$ & $\begin{array}{c}\text { Bulk Solute } \\
\text { Concentration (\%) }\end{array}$ & $\begin{array}{l}\text { Detection } \\
\text { Efficiency }\end{array}$ & $\begin{array}{c}\text { Lateral Scatter (Width of Gaussian } \\
\text { Applied to } X \text { and } Y \text { Coordinates) (nm) }\end{array}$ \\
\hline 1 & $0.5,1.0,1.5$ & $\sim 50$ and 100 & 1 & 100 & $\mathrm{NA}^{\mathrm{a}}$ \\
\hline 2 & $0.5,1.0,1.5$ & $\sim 50$ and 100 & 1 & 40 & NA \\
\hline 3 & $0.5,1.0,1.5$ & $\sim 50$ and 100 & 1 & 100 & 0.5 \\
\hline 4 & $0.5,1.0,1.5$ & $\sim 50$ and 100 & 1 & 40 & $0.5^{\mathrm{a}}$ \\
\hline 5 & $0.5,1.0,1.5$ & $\sim 50$ and 100 & 1 & 100 & 1.0 \\
\hline 6 & $0.5,1.0,1.5$ & $\sim 50$ and 100 & 1 & 40 & $1.0^{\mathrm{a}}$ \\
\hline
\end{tabular}

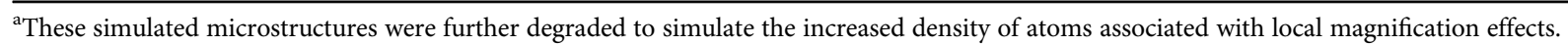




\section{Simulating the Increased Density of Atoms Associated with Local Magnification Effects}

Evaporation of a material composed of several phases with significant differences in field evaporation threshold induces an evolution of the steady state shape of the tip surface. The consequence is strong distortions of the ion trajectories named "local magnification" effects (Miller \& Hetherington, 1991). It changes both the $X, Y$ positions, and, to a lesser extent, the $Z$ positions. This effect can be highlighted by the presence of high- (or low)-density regions correlated with differences in local composition (Fig. 1).

When local magnification is observed, at the interface between phases, trajectory overlaps may be present. When the difference is too big, the phase of interest can be mixed completely with the surrounding matrix (ion crossing) (Miller \& Hetherington, 1991). The magnitude of ion crossing is difficult to assess. Limits can be determined based on the fact that small solute clusters can be identified. The modeling of the field evaporation of a thin needle at the atomic scale can also provide understanding of the origin of the bias in $3 \mathrm{D}$ reconstructions and quantitative information about their influence on chemical composition measurements (Blavette et al., 2001; Lefebvre et al., 2016).

Local magnification of solute clusters in RPV steels will result in compression or expansion in $X$ and $Y$ orientations, due to the fact that the solute atoms have different evaporation fields to that of $\mathrm{Fe}$, and thus clusters may appear ellipsoidal (elongated in the $Z$ direction) rather than spherical. For example, in the case of $\mathrm{Cu}$ clusters in an $\mathrm{Fe}$ matrix a compression of the clusters is observed.

The underlying physics and complexities of local magnification, and the associated resulting effect on atom positions in the reconstructed data, is extremely challenging to model. In this work, a pragmatic approach to consider the effect of local magnification in the simulated data files was taken and is outlined here.

Assuming cylindrical symmetry on the $Z$ axis of a single spherical cluster, the positions of atoms situated at the center of each cluster are not modified by local magnification effects. Similarly, far away from the cluster there is no effect of local magnification. These define the boundary conditions for the transformation (either from observed APT data to a more realistic picture of the underlying microstructure, or to transform perfect simulated data into a microstructure that is more representative of what would be observed by APT). The transformation, shown schematically in Figure 2, shows that it is necessary to consider both atoms within the solute cluster (compressed in APT data) and those in the adjacent matrix (more disparate in APT data).

Consider a solute cluster with radius $r$. As the cluster is slowly uncovered during an APT analysis, the radius of uncovered surface will increase to $\sim r$ and then decrease to 0 . The effective radius, as a function of depth $z$ in the cluster (where $-r<z<r$ ), is therefore given by

$$
R(z)=\sqrt{r^{2}-z^{2}} .
$$

The schematic in Figure 3 shows the displacement of each atom (i.e., the difference between where the atom is detected and where it would have been detected without local magnification). The displacement depends on the distance $(d)$ in the $X-Y$ plane to the center of the solute cluster and the
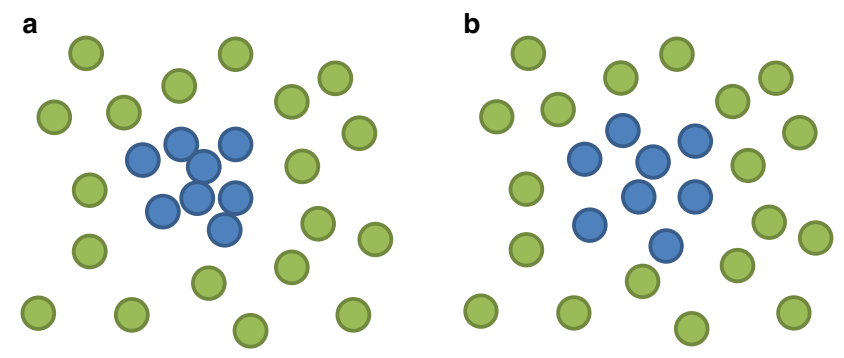

Figure 2. Schematic representation of (a) observed distribution of atoms from atom probe tomography analysis resulting from local magnification effects and (b) expected distribution without local magnification effects. Plan view ( $X-Y$ plane). "Blue" atoms have a lower evaporation field than the matrix.

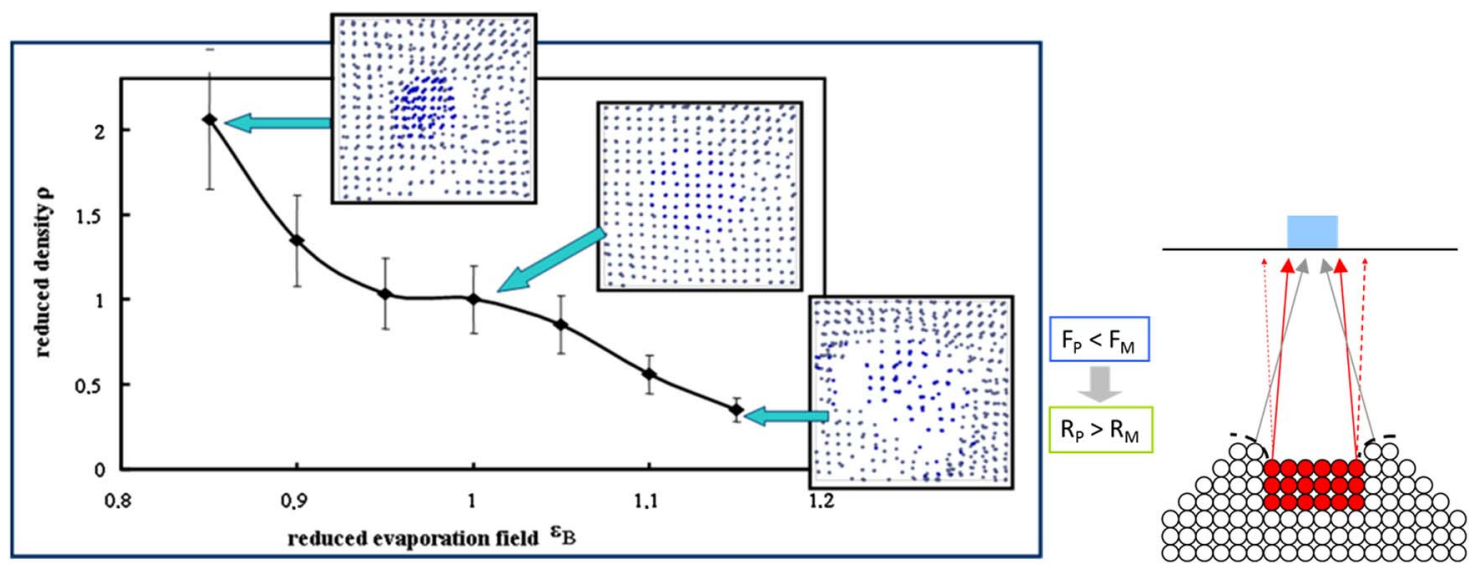

Figure 1. Local magnification effects for low evaporation field particle (left) or high evaporation field particle (right), and schematic drawing showing ion trajectories close to the surface of the evaporated phase (the red atoms have a lower evaporation field than the white). Note the presence of ion crossing at the interface. From Vurpillot et al. (2000). 


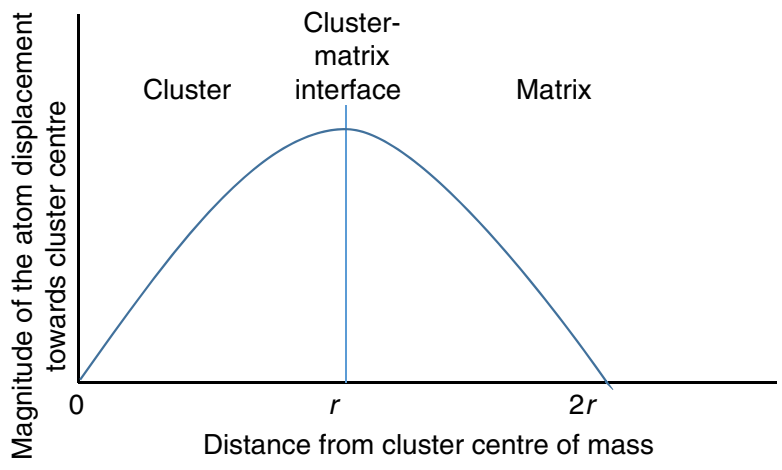

Figure 3. Schematic representation of displacement function in $X-Y$ plane to simulate the local magnification effect.

effective cluster radius $R(z)$. The function satisfies the boundary conditions, 0 at the center of each cluster and far away from the cluster. A positive gradient corresponds to a compression of the distances between atom positions, a negative gradient to an expansion. The "transformation" also ensures that the atoms preserve their relative positions to each other.

The exact form of the displacement function is not known and so a simple trial function was considered

$$
\Delta d \propto\left(k_{2} R(z)-|d|\right) d,
$$

where $k_{2}$ is a constant. In this case the maximum displacement will occur when $d=\frac{k_{2} R(z)}{2}$ and will equal 0 when $d=0$ or $d=k_{2} R(z)$. The maximum displacement is $\Delta d_{\max } \propto \frac{1}{4} k_{2}^{2} R(z)^{2}$. Hence, the shift can be normalized to have a maximum shift of $k_{1}$.

$$
\Delta d=k_{1} \frac{\left(k_{2} R(z)-|d|\right) d}{\frac{1}{4} k_{2}^{2} R(z)^{2}} .
$$

However, the maximum shift should increase with increasing $R(z)$. When $R(z)=0$, the shift should equal 0 , when $R(z)=r$ (the cluster radius) the shift should be a maximum. The maximum should depend on the size of the cluster. This can be achieved by multiplying by $\frac{R(z)}{r}$ as $(R(z)$ can have any value between 0 and $r$ ). Thus, a better model for the displacements is

$$
\begin{aligned}
\Delta d & =\frac{\left(k_{2} R(z)-|d|\right) d}{\frac{1}{4} k_{2}^{2} R(z)^{2}} \cdot \frac{R(z)}{r} \cdot(k-1) R(z) \\
& =\frac{\left(k_{2} R(z)-|d|\right) d}{\frac{1}{4} k_{2}^{2} r} \cdot(k-1),
\end{aligned}
$$

with $k$ defined as a compression coefficient $\left(k_{1}+1\right)$. Thus with $k=1.1$ (a compression coefficient of $10 \%$ ), $\Delta d_{\max }=$ $0.1 R(z)$, corresponding to a displacement toward the cluster center making the cluster $10 \%$ smaller in the $X-Y$ plane.

With $k_{2}=2,\left[d_{\max }\right.$ is set to be $\left.2 R(z)\right]$ the equation simplifies to

$$
\Delta d=\frac{(2 R(z)-|d|) d}{r} .(k-1) .
$$

Tests were performed to determine how robust the approach adopted is. APT data from an irradiated RPV steel
Table 2. Density of Atoms in Clusters in (i) Atom Probe Tomography (APT) Data and (ii) APT Data Following Simulation to Reverse the Effect of Local Magnification.

\begin{tabular}{lcc}
\hline & \multicolumn{2}{c}{ Atoms $/ \mathrm{nm}^{3}$ in Solute Clusters } \\
\cline { 2 - 3 } & $\begin{array}{c}\text { As } \\
\text { Reconstructed } \\
\text { APT Data }\end{array}$ & $\begin{array}{c}\text { Magnification } \\
\text { Transformation with } \\
k_{1}=1.25\end{array}$ \\
Radius (nm) & 36 & 36 \\
Entire reconstructed & & \\
$\quad$ volume & $49.0 \pm 10.4$ & $39.2 \pm 11.5$ \\
$0.75-1.25$ & $58.1 \pm 13.1$ & $33.9 \pm 9.3$ \\
$1.25-1.75$ & $65.8 \pm 9.7$ & $39.3 \pm 7.2$ \\
$1.75-2.25$ & $66.4 \pm 12.3$ & $40.6 \pm 5.7$ \\
$2.25-2.75$ & &
\end{tabular}

containing a range of solute cluster sizes (0-5 $\mathrm{nm}$ diameter) was selected. The MSM method was used to identify atoms belonging to each cluster. Each cluster was then analyzed to determine the associated center of mass and principle axes of the best fit ellipsoid. The observed density of atoms in the solute enriched clusters was then determined by dividing the number of atoms within the best fit ellipsoid by the ellipsoid volume. The atoms included in these calculations can include both those assigned as cluster atoms and also matrix atoms. The resultant density data were analyzed as a function of cluster size. The transformation was applied, selecting a single value of $k_{1}$ to spread out atoms within the clusters to a density consistent with the rest of the reconstructed data. The transformed data were re-analyzed, and the observed densities of atoms in each cluster size measured. The experimental data set selected was from Ringhals NPP and has been published (Styman et al., 2015).

The results show that the apparent density of atoms in clusters in this APT data are 60-70\% greater than what would be expected from a typical steel with $37 \%$ detection efficiency. The same value of $k$ was applied to all clusters in the data set irrespective of diameter. Table 2 and Figure 4 show that a single value of $k$ reduced the density to close to the average for the entire reconstructed volume $\left(36\right.$ atoms $\left./ \mathrm{nm}^{3}\right)$. A single value of $k$ can be used for a range of cluster sizes.

\section{Analysis of Solute Clusters}

The MSM is one of the most commonly used cluster identification algorithms. Several authors have provided detailed explanations of its strengths and weaknesses and made recommendations regarding how best to optimize the parameters (Cerezo \& Davin, 2007; Kolli \& Seidman, 2007; Styman et al., 2013; Williams et al., 2013; Jägle et al., 2014).

A second method, named "Isoposition method" (IPM) was also used to identify clusters. The principle of this method which is based on solute concentration criteria is described elsewhere (Lefebvre et al., 2016). Roughly, a 3D 


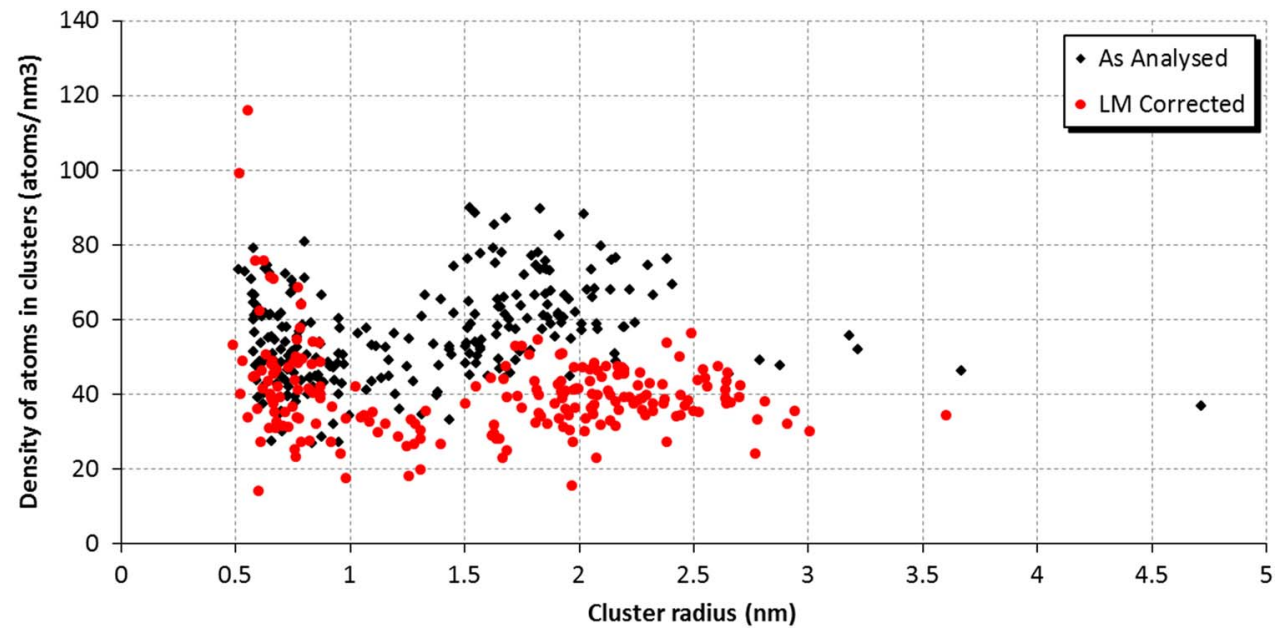

Figure 4. Densities of atoms in clusters in the as reconstructed data and after applying local magnification transform with $k=1.25$.

concentration map is used to define the local concentration associated with the position of each atom inside the volume. A concentration threshold $\left(C_{\min }\right)$ is then applied on all positions to select and filter out atoms that will be considered to identify the clusters. If the filtered atoms are separated by a distance smaller than a given value $\left(d_{\max }\right)$, they belong to the same cluster. Only clusters containing a minimum number of solute atoms $\left(N_{\min }\right)$ are considered. The values of the user defined parameters are determined by comparing the simulated data files with randomized ones (in that case, the chosen parameters should not result in cluster detection). It is important to note that after this identification step, if two clusters are identified as a unique one or if a cluster is cut in two parts during identification, they are separated or merged after visual inspection. Then an erosion is applied to the clusters to remove interface atoms. The interface is then located at the half-maximum of the cluster core composition. The cluster size is calculated after erosion. The given cluster composition is the core composition.

Both MSM and IPM rely upon user-defined parameters which can strongly influence the results. For this work the following parameters were used for MSM and IPM:

\section{- MSM}

o $D_{\max }=0.4 \mathrm{~nm}$,

o $N_{\text {min }}=9$ or 22 (equivalent to the number of solutes in a $0.5 \mathrm{~nm}$ cluster of $50 \%$ solute with 40 or $100 \%$ detection efficiency, respectively),

o $L=e=0.3 \mathrm{~nm}$ (set to be $0.1 \mathrm{~nm}$ less than $D_{\max }$, previous analyses have shown results do not depend strongly on choice of $L$ or $e$ : Hyde et al., 2011).

- IPM

o $C_{\text {threshold }}=5.5-5.7 \%$ : set to the value for which the concentration histogram of randomized data set is negligible $(<0.1 \%)$,

o $D_{\max }=0.5 \mathrm{~nm}$ (small but voxels always contain a few atoms), o $N_{\min }=6-10$ : set so no clusters identified in a randomized volume with $C_{\text {threshold, }}$

o $D_{\text {erosion }}=0.4-0.8 \mathrm{~nm}$ : set at half maximum of the erosion profile.

The microstructure simulations were analyzed using both the MSM and IPM. The precise parameters used to seed the microstructural simulations were then revealed and an assessment of the accuracy of the two analysis methods performed.

For both methods the cluster radii are given by average of the Guiner radius, $R_{G}$ which is the gyration radius multiplied by $\sqrt{ }(5 / 3)$, to give the true radius assuming spherical clusters of uniform atom density. Cluster compositions are reported as the average of the compositions of individual clusters.

\section{Results}

Each simulated microstructure contained 108 solute clusters (although this information was not provided to the scientists analyzing the simulated data). The number of clusters detected by MSM and IPM is presented in Figure 5. All the clusters were detected in the microstructures that had not been degraded (100\% detection efficiency, perfect lateral resolution) or had been degraded by either a reduction in detection efficiency (to $40 \%$ ) or by the introduction of lateral scatter $(2 \sigma=0.5 \mathrm{~nm}$ or $1 \mathrm{~nm})$. However, the combination of both a reduced detection efficiency and loss of lateral resolution did impact the cluster detectability. In these situations, for the $0.5 \mathrm{~nm}$ clusters, the MSM was not able to accurately distinguish between clusters which were seeded and those which were the result of random statistical fluctuations (sometimes referred to as background or random clusters). In this instance the IPM was more efficient at detecting the $0.5 \mathrm{~nm}$ clusters than the MSM method.

Figure 6 shows the observed cluster sizes determined using the MSM and IPM methods. The error bars represent the range of cluster sizes shown. The analysis of perfect data 


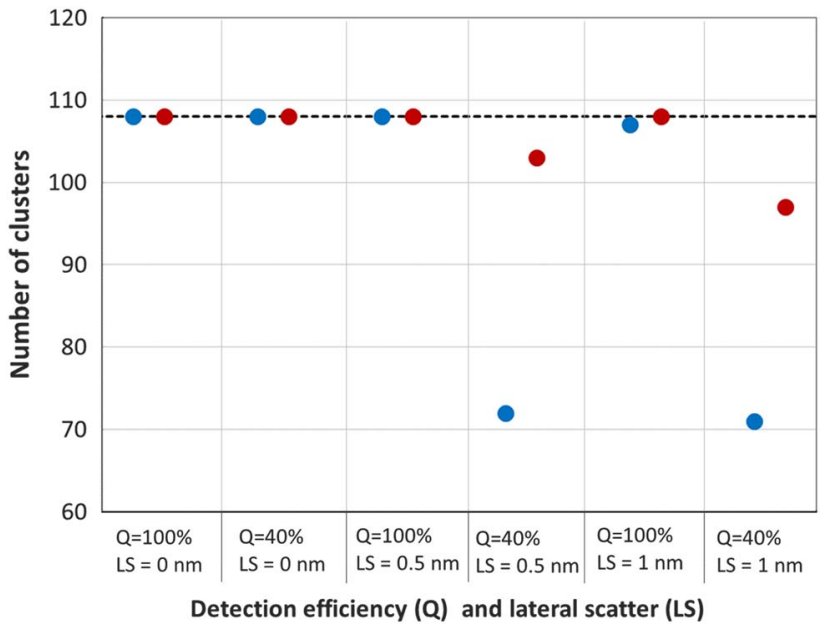

Figure 5. Number of clusters identified using maximum separation method (blue) and isoposition method (red) as a function of detection efficiency $(Q)$ and lateral scatter (LS). The dotted line is the true number of clusters.

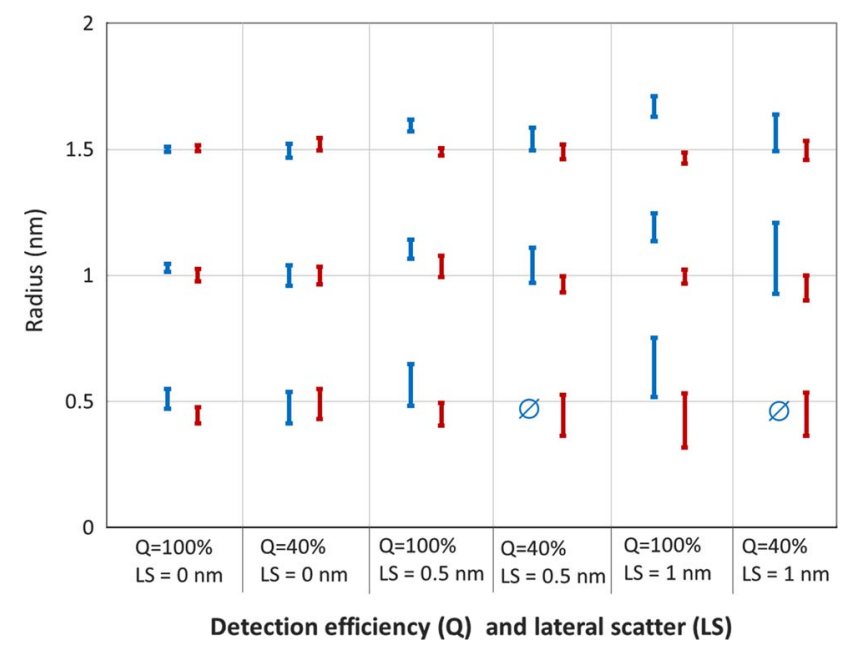

Figure 6. Observed cluster sizes determined using the maximum separation method (blue) and isoposition method (red).

(100\% detection efficiency and no lateral scatter) shows excellent agreement between the two methodologies. A degradation in either the detection efficiency or lateral resolution increases the range of cluster sizes observed but the results are still self-consistent. The most challenging scenario involves identifying clusters with a radius of $\sim 0.5 \mathrm{~nm}$, degraded by the imperfect detection efficiency and lateral scatter (made even more difficult if clusters only contain 50\% solute). The IPM was able to detect some of these, but the MSM was not able to distinguish these from random solute fluctuations.

Figure 7 shows the observed mean cluster compositions determined by the MSM and IPM. The data have been split by cluster size (different colors) and nominal solute level (100 or $\sim 50 \%)$. An examination of the results from perfect data (high detection efficiency and perfect lateral resolution) shows that both analysis techniques accurately identify the larger clusters (radius $\geq 1 \mathrm{~nm}$ ). However, the compositions of

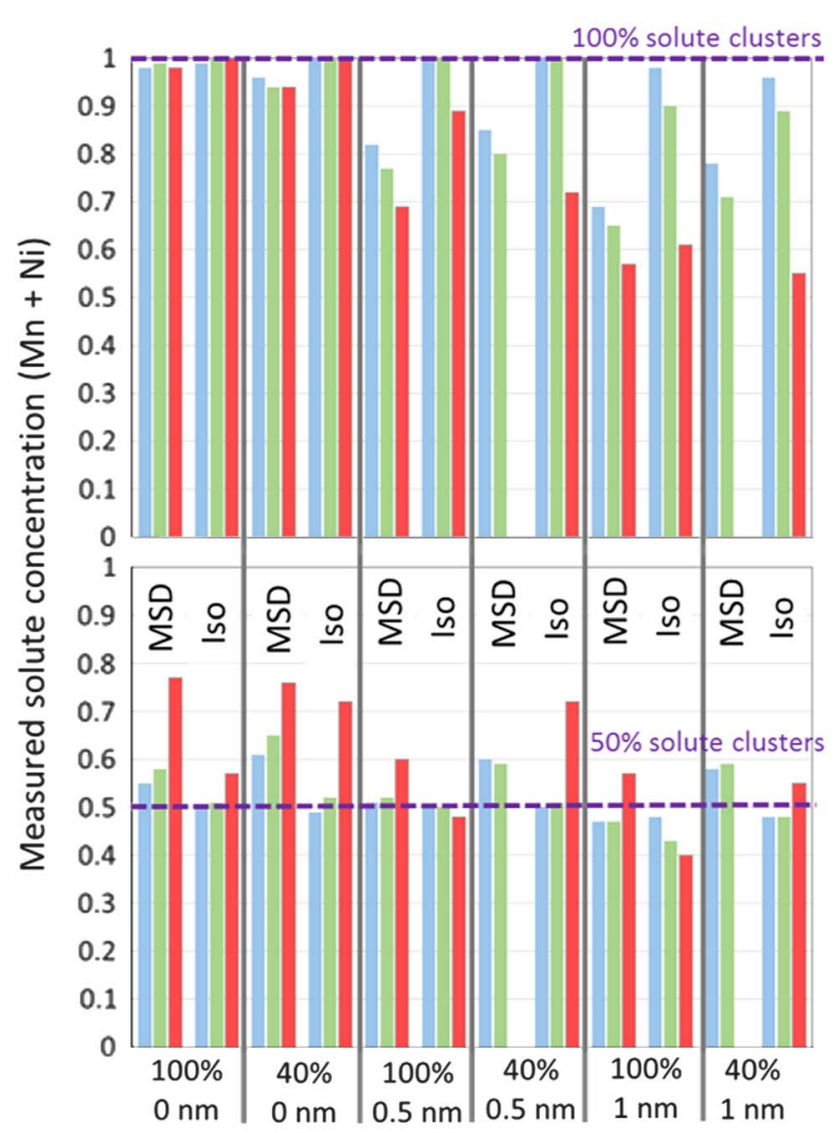

Detection efficiency and lateral resolution

Figure 7. Observed compositions using maximum separation method (MSD in figure) and isoposition method (Iso in figure) for clusters containing 100 and $\sim 50 \%$ solute atoms. The colors relate to the cluster radii $-\sim 1.5 \mathrm{~nm}$ (blue), $\sim 1 \mathrm{~nm}$ (green), and $\sim 0.5 \mathrm{~nm}$ (red). Note that for a detection efficiency of $40 \%$ and a degraded lateral resolution, the clusters of about $0.5 \mathrm{~nm}$ with $\sim 50$ and $\sim 100 \%$ solutes cannot be distinguished when detected.

the smaller clusters (radius $\sim 0.5 \mathrm{~nm}$ ) can be overestimated using MSM. If the lateral resolution is degraded, the observed $\mathrm{Fe}$ content increases and this is particularly noticeable for the smallest clusters, where the surface atoms have an increasingly significant influence on the observed overall cluster composition. If the lateral resolution is degraded and the detection efficiency reduced to $40 \%$, it becomes increasingly difficult to identify the smallest (radius $=0.5 \mathrm{~nm}$ ) clusters. More of these clusters are detectable using the IPM than the MSM, but the estimated cluster compositions were $\sim 50 \%$ solute irrespective of their true solute composition ( 50 or $100 \%)$.

The results indicate that clusters with a radius of $\sim 0.5 \mathrm{~nm}$ are near the cluster detectability limit using current APT (although it should be noted that compositional variations on this scale can be detected by alternative statistical techniques). The results for the clusters with a radius of $\sim 1 \mathrm{~nm}$ (green) are very similar to those with a radius of $\sim 1.5 \mathrm{~nm}$ (blue) demonstrating the robustness of both methodologies used for detecting larger clusters. 
a

Compressed clusters $n$ Non-compressed clusters
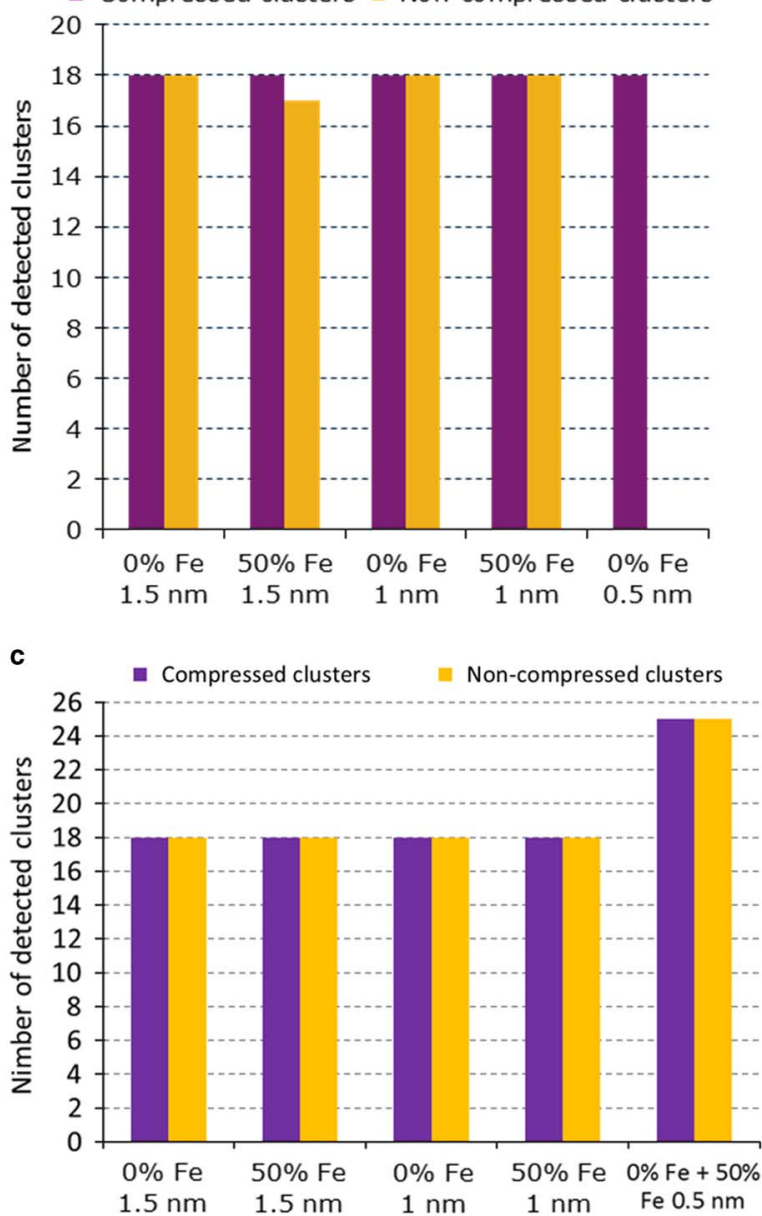

b

Compressed clusters Non-compressed clusters

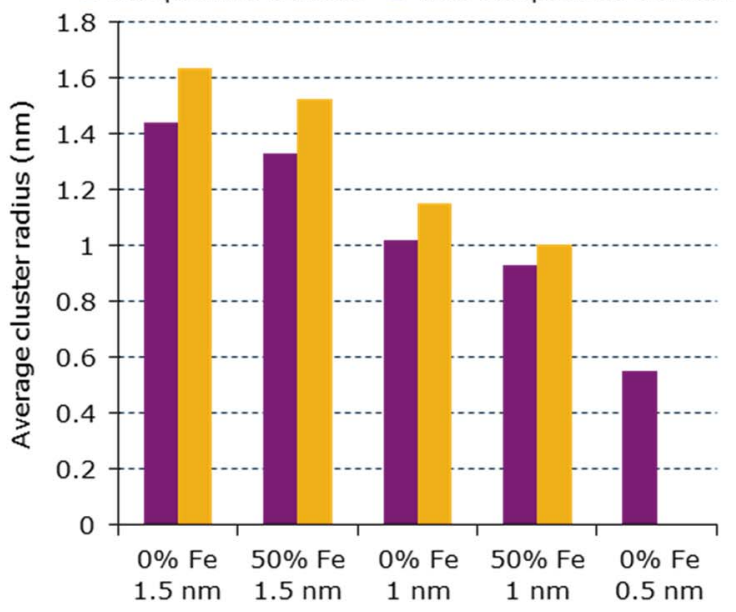

d

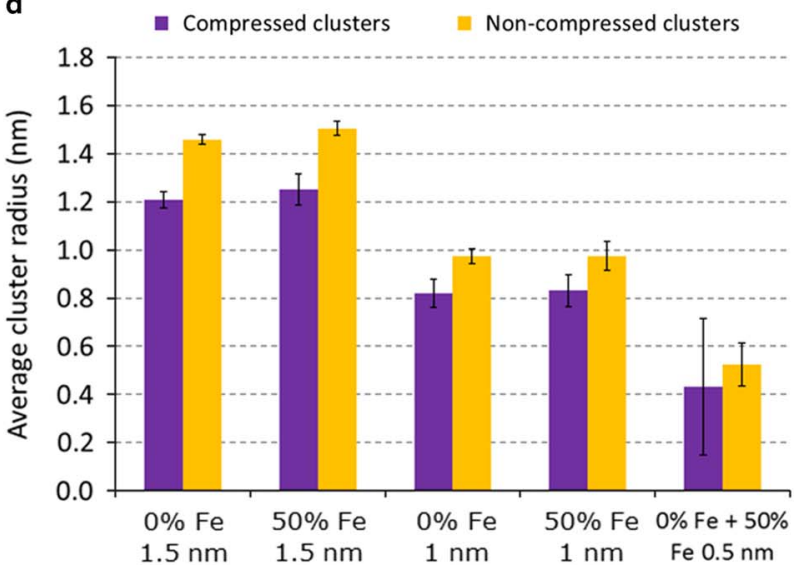

Figure 8. Simulation 6 analyzed with and without local Mag simulation using the maximum separation method and the isoposition method. a,c: Effect of local magnification on the number of clusters detected (b,d) Effect of local magnification on the measured cluster radius (Comparison between cluster sizes with and without the simulation of local magnification).

In real data sets, local magnification can occur. It compresses solute clusters in the $X$ and $Y$ directions which can affect detection of clusters and the observed cluster sizes (in contrast to the positioning uncertainty). Therefore, local magnification was applied to the simulated microstructure in which the detection efficiency had been set to $40 \%$ and a Gaussian scatter of width $1 \mathrm{~nm}$ had been added to the $X$ and $Y$ coordinates.

Analysis of this new data set, using MSM, identified 90 clusters in comparison with the 71 previously identified. In terms of cluster detection, the effect of local magnification means that the $0.5 \mathrm{~nm}, 100 \%$ solute clusters can now be separated from the random solute fluctuations using MSM. As was the case previously (before the application of local magnification effects) the $0.5 \mathrm{~nm}, 50 \%$ solute clusters still cannot be separated from the random solute fluctuations. The number of clusters identified with IPM method remains constant (97 with or without local magnification) (Figure 8).

The magnitude of the effect of local magnification on size measurement is also shown in Figure 8. Obviously, both IPM and MSM give a lower Guinier radius if there is local magnification. In such cases, the Guinier radius is probably not the best parameter to quantify the cluster size.

\section{Discussion}

The results are summarized in Table 3 . Not surprisingly both methodologies worked extremely well with perfect data. Both MSM and IPM worked well when a single degradation phenomenon was introduced. Results from the IPM are affected less by the introduction of a lateral scatter to the atom positions than for the MSM. This is evident in the slightly more accurate estimates of cluster sizes and compositions. Aside from the detection method itself, these more accurate estimates could be due to the fact that (i) an erosion of the interface atoms is performed after cluster identification with IPM and (ii) the cluster composition is the core composition. The scatter does increase the extent of the clusters and this is detected by the MSM method. In contrast, the IPM method followed by erosion tends to provide a slightly lower estimate of cluster sizes. 
Table 3. Summary of Observations.

\begin{tabular}{|c|c|c|}
\hline & MSM & IPM \\
\hline Perfect data & $\begin{array}{l}\text { All clusters identified } \\
\text { Solute content of dilute smallest clusters } \\
\text { overestimated }\end{array}$ & All clusters identified and correctly characterized \\
\hline $\begin{array}{l}\text { Imperfect detection } \\
\text { efficiency }\end{array}$ & $\begin{array}{l}\text { All clusters identified } \\
\text { Solute content of dilute smallest clusters } \\
\text { overestimated }\end{array}$ & $\begin{array}{l}\text { All clusters identified } \\
\text { Solute content of dilute smallest clusters } \\
\text { overestimated }\end{array}$ \\
\hline $\begin{array}{l}\text { Perfect detection but } \\
\text { degraded lateral } \\
\text { resolution }\end{array}$ & $\begin{array}{l}\text { All, or nearly all, clusters identified } \\
\text { Lateral scatter increases apparent cluster size } \\
\text { Solute content of concentrated clusters } \\
\text { underestimated }\end{array}$ & $\begin{array}{l}\text { All clusters identified } \\
\text { Good estimates of cluster sizes and solute content } \\
\text { excepted for pure small }(0.5 \mathrm{~nm}) \text { clusters } \\
\text { (overestimation of } \mathrm{Fe} \text { content) }\end{array}$ \\
\hline $\begin{array}{l}\text { Imperfect detection } \\
\text { efficiency and } \\
\text { degraded lateral } \\
\text { resolution }\end{array}$ & $\begin{array}{l}\text { Significant fraction of smallest clusters not } \\
\text { identified } \\
\text { Increasingly difficult to determine cluster sizes } \\
\text { accurately } \\
\text { The observed compositions of dilute clusters are } \\
\text { reasonable but the compositions of the more } \\
\text { concentrated clusters are underestimated }\end{array}$ & $\begin{array}{l}\text { A small fraction of smallest clusters not identified. } \\
\text { More scatter observed on cluster sizes, but the mean } \\
\text { cluster sizes are approximately correct } \\
\text { The observed compositions of dilute clusters are } \\
\text { reasonable but the compositions of the more } \\
\text { concentrated clusters are underestimated } \\
\text { Not possible to distinguish dilute and concentrated } \\
\text { smallest clusters in terms of composition }\end{array}$ \\
\hline
\end{tabular}

MSM, maximum separation method; IPM, Isoposition method.

Analysis becomes more challenging when both the imperfect detection efficiency and degradation of lateral resolution are simulated. However, even under these conditions, both methods worked extremely well for clusters with radii $\gtrsim 1 \mathrm{~nm}$. Accurate characterization of the smallest clusters $(0.5 \mathrm{~nm}$ radius) proved more challenging. The MSM method failed to separate a significant number of these clusters from random solute fluctuations. The IPM proved to be more effective at identifying the presence of these clusters, but it was not possible to distinguish between the concentrated $(100 \%$ solute) clusters and the dilute (containing 50\% solute).

Several reasons can explain these observations. First, in terms of statistics, the smallest clusters contain so few solute atoms that their definition is strongly influenced by surface effects. So, strong difference in composition can result from the interface definition. Including an erosion procedure, can strongly modify the results in this case. Second, as it is shown in Figure 9, the lateral scatter (without considering any trajectory aberration due to difference in field evaporation) can result in the introduction of a significant level of matrix atoms in the cluster core. So, a concentrated small solute cluster appears to be significantly diluted due to the lateral scatter. Thus, both MSM and IPM will detect a high level of Fe in the smallest clusters.

In real APT data, local magnification will result in an increase in the density of atoms in the clusters. Counterintuitively, this can actually aid identification of solute clusters with the MSM as it is predicated on finding regions in which the solute atoms are more closely spaced. On the contrary, local magnification has no influence on the cluster detection using IPM. This is not surprising as the IPM is based on concentration threshold and not on distance between atoms. As explained previously, the algorithm applied to reproduce local magnification results in a change in the local atom density, but not in local concentration as trajectory overlaps were not simulated.

The limitations reported here in the methods of analysis of APT data in terms of correct detection (or not) of clusters below a certain size, over- or underestimation of size and concentration of solutes, etc. should be used also to provide atomistic modelers with indications, and possibly protocols, about how to analyze the results of simulations for a fair comparison.

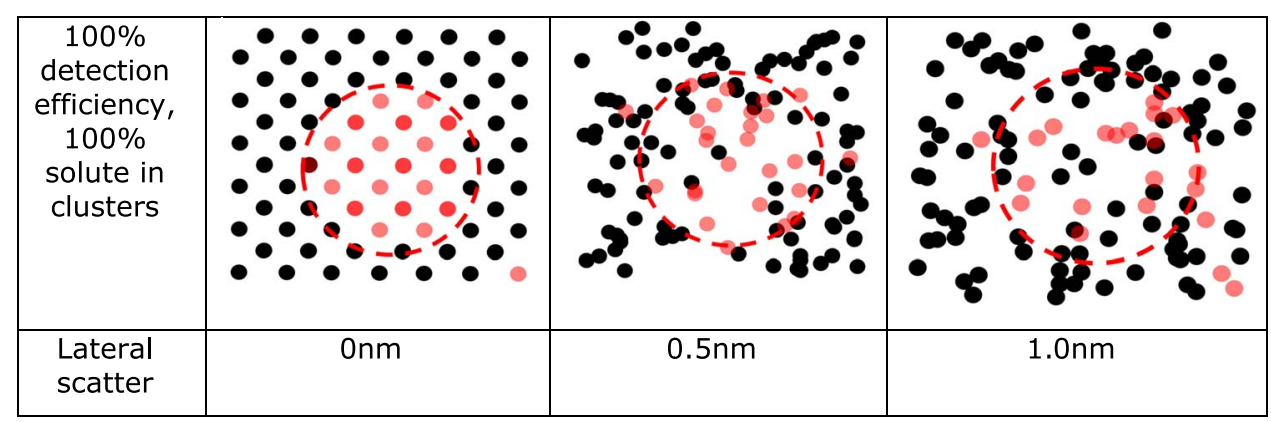

Figure 9. Effect of lateral scatter on the definition of a solute cluster. 
Atomistic simulations contain the position and type of all atoms, therefore, an analysis of density, size, and composition applied directly to simulated results cannot be directly compared with APT results. The data (atomic positions and types) should at least be treated similarly to what has been done in this work (Methodology section) in terms of lateral resolution and detection efficiency, so as to mimic the effect of APT. Indications about the best way to do so, depending on the APT technique, should be given by experimentalists. Moreover, the limitations of the analysis methods (e.g., the overestimation of the size of small clusters using MSM, or its underestimation using IPM) should be used as a criterion to judge the comparison between simulation and experiments. Ideally, precise protocols should be deduced on how to treat simulation data, in order for the comparison with APT experiments to be performed on equal footing.

\section{CONCLUSIONS}

The aim of this work was to provide insight into the limitations and uncertainties associated with reported APT data, which would be useful both to the atom probe community and also to modelers. Simulations of microstructural data, which take into account estimates of common experimental artifacts associated with APT (reduced detection efficiency, positioning uncertainty, and local magnification effects), were performed. A simple method for modeling local magnification effects was proposed and it was shown to be effective in accounting for the observed increased density of atoms in solute clusters in experimental data.

The resulting simulated microstructures have been analyzed using two algorithms, the MSM and the IPM. The results show that for the identification of clusters $\gtrsim 1 \mathrm{~nm}$, both MSM and IPM work extremely well, provided that the parameters are carefully chosen. Detection of clusters with a radius of $\sim 0.5 \mathrm{~nm}$ is possible, but care is needed interpreting quoted compositions. Further work on core shell structured clusters is underway and will provide additional insight on the capability of methodologies used to characterize clusters observed in atom probe data.

Recommendations will be developed which, if adopted, will enable inter-comparison of results between different labs, enabling trends in microstructural development to be more readily observed, and provide information that can be directly used to support calibration of models from the modeling community.

\section{ACKNOWLEDGMENTS}

This work was part-funded under the EU FP7 NUGENIA+ project. Grant number 604965 .

\section{REFERENCES}

Auger, P., Pareige, P., Akamatsu, M. \& Blavette, D. (1995). APFiM investigation of clustering in neutron-irradiated $\mathrm{Fe}-\mathrm{Cu}$ alloys and pressure vessel steels. J Nucl Mater 225, 225-230.
Blavette, D., Vurpillot, F., Pareige, P. \& Menand, A. (2001). A model accounting for spatial overlaps in $3 \mathrm{D}$ atom-probe microscopy. Ultramicroscopy 89, 145-153.

Carter, R.G., Soneda, N., Dohi, K., Hyde, J.M., English, C.A. \& SERVER, W.L. (2001). Microstructural characterization of irradiation-induced $\mathrm{Cu}$-enriched clusters in reactor pressure vessel steels. J Nucl Mater 298, 211-224.

Cerezo, A. \& Davin, L. (2007). Aspects of the observation of clusters in the 3-dimensional atom probe. Surf Interface Anal 39, 184-188.

Gault, B., Moody, M.P., Cairney, J.M. \& Ringer, S.P. (2012). Atom Probe Microscopy. New York: Springer.

Heinrich, A., Al-Kassab, T. \& KirChHeim, R. (2003). Investigation of the early stages of decomposition of $\mathrm{Cu} 0.7$ at.\% $\mathrm{Fe}$ with the tomographic atom probe. Mater Sci Eng A353, 92-98.

Huang, H., Radiguet, B., Todeschini, P., Rainasse, C., Clémendot, F. \& Pareige, P. (2014). Influence of $\mathrm{Cu}$ and $\mathrm{Ni}$ levels on the microstructural evolution of French reactor pressure vessel steels. Proc Fontevraud 8.

Hyde, J.M. \& English, C.A. (2001). An analysis of the structure of irradiation induced $\mathrm{Cu}$-enriched clusters in low and high nickel welds. Mat Res Soc Symp. Proc 650, R6.6.1-R6.6.12.

Hyde, J.M., Marquis, E.A., Wilford, K.B. \& Williams, T.J. (2011). A sensitivity analysis of the maximum separation method for the characterisation of solute clusters. Ultramicroscopy 111, 440-447.

Jägle, E.A., ChoI, P.-P. \& RaAbe, D. (2014). The maximum separation cluster analysis algorithm for atom-probe tomography: Parameter determination and accuracy. Microsc Microanal 20, 1662-1671.

Kolli, R.P. \& SEIDMAn, D.N. (2007). Comparison of compositional and morphological atom-probe tomography analyses for a multicomponent $\mathrm{Fe}-\mathrm{Cu}$ steel. Microsc Microanal 13, 272-284.

Lefebvre, W., Vurpillot, F. \& Sauvage, X. (2016). Atom Probe Tomography - Put Theory Into Practice. Elsevier.

MARQuis, E.A. \& Hyde, J.M. (2010). Atomic scale analysis of solute behaviours by atom-probe tomography. Mater Sci and Eng $R$ Reports 69, 37-62.

Miller, M.K. (2000). Atom Probe Tomography Analysis at the Atomic Level. New York: Kluwer Academic/Plenum Publishers.

Miller, M.K., Cerezo, A., Hetherington, M.G. \& Smith, G.D.W. (1996). Atom Probe Field Ion Microscopy. Oxford: Oxford Science Publications.

Miller, M.K. \& Hetherington, M.G. (1991). Local magnification effects in the atom probe. Surf Sci 246, 442-449.

Miller, M.K. \& Russell, K.F. (2007). Embrittlement of RPV steels: An atom probe tomography perspective. J Nucl Mater 371, 145-160.

Miller, M.K., Russell, K.F., KociK, J. \& Keilova, E. (2000). Embrittlement of low copper VVER 440 surveillance samples neutron-irradiated to high fluences. J Nucl Mater 282, 83-88.

Miller, M.K., Russell, K.F., Sokolov, M.A. \& Nanstad, R.K. (2007). APT characterization of irradiated high nickel RPV steels. J Nucl Mater 361, 248-261.

Morley, A., Sha, G., Hirosawa, S., Cerezo, A. \& Smith, G.D.W. (2009). Determining the composition of small features in atom probe: BCC Cu-rich precipitates in an Fe-rich matrix. Ultramicroscopy 109, 535-540.

ODETTE, G.R. (1995). Radiation induced microstructural evolution in reactor pressure vessel steels. Mat Res Soc Symp Proc 373, 137-148. 
Pareige, P., Stoller, R.E., Russell, K.F. \& Miller, M.K. (1997). Atom probe characterization of the microstructure of nuclear pressure vessel surveillance materials after neutron irradiation and after annealing treatments. J Nucl Mater 249, 165-174.

Radiguet, B., Pareige, P. \& Barbu, A. (2009). Irradiation induced clustering in low copper or copper free ferritic model alloys. Nucl Instrum Methods Phys Res B 267, 1496-1499.

Styman, P.D., Hyde, J.M., Parfitt, D., Wilford, K., Burke, M.G., ENGLish, C.A. \& EFsing, P. (2015). Post-irradiation annealing of $\mathrm{Ni}-\mathrm{Mn}$-Si-enriched clusters in a neutron-irradiated RPV steel using atom probe tomography. J Nucl Mater 459, 127-134

Styman, P.D., Hyde, J.M., Wilford, K. \& Smith, G.D.W. (2013). Quantitative methods for the APT analysis of thermally aged RPV steels. Ultramicroscopy 132, 258-264.
Takeuchi, T., Kuramoto, A., Kameda, J., Toyama, T., Nagai, Y., Hasegawa, M., Ohkubo, T., Yoshite, T., Nishiyama, Y. \& Onizawa, K. (2010). Effects of chemical composition and dose on microstructure evolution and hardening of neutron irradiated reactor pressure vessel steels. J Nucl Mater 402, 93-101.

Vurpillot, F., Bostel, A. \& Blavette, D. (2000). Trajectory overlaps and local magnification in three-dimensional atom probe. Appl Phys Lett 76, 3127-3129.

Wells, P.B., Yamamoto, T., Miller, B., Milot, T., Cole, J., Wu, Y. \& OdetTe, G.R. (2014). Evolution of manganese-nickel-silicondominated phases in highly irradiated reactor pressure vessel steels. Acta Mater 80, 205-219.

Williams, C.A., Haley, D.J., Marquis, E.A., Smith, G.D.W. \& Moody, M.P. (2013). Defining clusters in APT reconstructions of ODS steels. Ultramicroscopy 132, 271-278. 